\title{
Evaluación mediante una encuesta del grado de conocimiento genético en la práctica clínica de oftalmólogos
}

\section{Evaluation by means of a survey about genetic knowledge in the clinical practice of ophthalmologists}

\author{
Bethania López-Star ${ }^{1 *}$, José F. Pérez-Pérez², Priscila Sánchez-Moreno ${ }^{3}$ y Luis A. Ochoa-Ramírez ${ }^{3}$ \\ ${ }^{1}$ Área de Teleoftalmología y Epidemiología; ${ }^{2}$ Oftalmología Pediátrica y Estrabismo; ${ }^{3}$ Unidad de Telemedicina. Instituto Mexicano de Oftalmología, \\ Querétaro, Querétaro, México
}

\section{Resumen}

Objetivo: Describir los resultados de una encuesta realizada a oftalmólogos pertenecientes a la Asociación Mexicana de Oftalmología Pediátrica (AMOP), a la Sociedad Latinoamericana de Oftalmología Pediátrica (SOPLA) y a la Sociedad Panamericana de Retinopatía del Prematuro (SPROP), sobre el abordaje de patologías de probable origen genético, así como sobre el uso de pruebas diagnósticas, el conocimiento actual y el futuro interés de aprendizaje. Método: Estudio observacional y descriptivo. Se aplicó un cuestionario mediante una plataforma electrónica, el cual consta de 24 preguntas, que fue compartido por medio de invitaciones electrónicas. Se realizó un análisis estadístico descriptivo de las variables de interés y para cada una se obtuvieron las prevalencias para la muestra total; los resultados se expresaron como porcentajes. Resultados: De los 100 médicos entrevistados, el 73\% reportaron no realizar un abordaje diagnóstico con enfoque genético. Patologías como el nistagmo, la catarata juvenil/congénita y el glaucoma congénito se asociaron en un $77 \%$, un $95 \%$ y un $94 \%$, respectivamente, con un componente genético en su etiología. El 55\% de los médicos reportaron no estar familiarizados con técnicas de diagnóstico genético. El interés de los médicos para aprender sobre genética aplicada en su práctica clinica es alto (64\%), mostrando un interés especial en la secuenciación completa del exoma. Conclusiones: En la práctica clínica de los médicos encuestados existe una deficiencia en el reconocimiento de patologías de origen genético, así como en su abordaje diagnóstico.

Palabras clave: Conocimiento. Diagnóstico genético. Encuesta. Oftalmología pediátrica. Patología oftálmica. Técnicas diagnósticas.

\section{Abstract}

Purpose: To describe the results obtained by the application of a survey to a group of ophthalmologists who are members of the Asociación Mexicana de Oftalmología Pediátrica (AMOP), Sociedad Latinoamericana de Oftalmología Pediátrica (SOPLA) and Sociedad Panamericana de Retinopatía del Prematuro (SPROP), about practice patterns, diagnostic techniques, current knowledge, and future educational interests about ophthalmic genetic disorders. Methods: Cross-sectional, descriptive study. An online survey of 24 questions was shared through electronic invitations. Statistical analysis used descriptive

\section{Correspondencia:}

*Bethania López-Star

Avda. Estadio, s/n

Colinas de Cimatario, Centro Sur

C.P. 76090, Santiago de Querétaro, $\quad$ Fecha de recepción: 05-08-2020

Querétaro, México $\quad$ Fecha de aceptación: 16-12-2020

Disponible en internet: 05-05-2021

E-mail: bethania.lopez@imoiap.edu.mx

DOI: 10.24875/RMO.M21000154

Rev Mex Oftalmol. 2021;95(3):101-106

un articulo open access bajo la (http://creativecommons.org/licenses/by-nc-nd/4.0/). 
statistics for the main variables, obtaining prevalences and expressing the results as percentages. Results: From the 100 physicians interviewed, $73 \%$ reported not carrying out genetic tests on patients with probable genetic eye disorders. Conditions as nystagmus, juvenile/congenital cataract and congenital glaucoma, were associated in $77 \%, 95 \%$ and $94 \%$, respectively, to have a genetic component in their etiologies. Fifty-five percent of physicians reported not being familiar with genetic diagnostic techniques. The interest of physicians to learn about applied genetics in their clinical practice is high (64\%), showing special interest in whole exome sequencing. Conclusions: In the clinical practice of physicians, there is a deficien$c y$ in the recognition of diseases with a genetic origin, as well as in their diagnostic approach.

Key words: Knowledge. Genetic testing. Survey. Pediatric ophthalmology. Eye disorders. Genetic techniques.

\section{Introducción}

La medicina está avanzando rápidamente dentro del ámbito de la genómica, y el ojo tiene un papel principal en la terapia génica. En la actualidad se conocen unas 4000 enfermedades o síndromes genéticos que afectan al hombre, de los cuales un tercio presentan involucro ocular. En el área de la oftalmología se ha logrado, en las últimas tres décadas, el mapeo de más de 300 genes y variantes causantes de enfermedades oculares inflamatorias y hereditarias ${ }^{1,2}$.

Las causas más importantes de ceguera en la infancia en los países con altos ingresos son el déficit visual cerebral y las anormalidades del nervio óptico, mientras que la retinopatía del prematuro y la catarata son las causas de ceguera prevenibles más frecuentes. Un estudio realizado en población inglesa en el año 2006 documenta una prevalencia de catarata congénita de 1-3 por 10,000 nacidos vivos, de retinitis pigmentosa de 1 por 4000 nacidos vivos, de retinoblastoma de 1 por 23,000 nacidos vivos y de aniridia de 1.8 por 100,000 nacidos vivos ${ }^{2}$. Más del $60 \%$ de los casos de ceguera en la infancia son causados por enfermedades heredadas, como catarata congénita, glaucoma congénito, degeneraciones retinianas, atrofia de nervio óptico, etc. Así mismo, el $40 \%$ de los pacientes con ciertos tipos de estrabismo cuentan con antecedentes familiares ${ }^{3}$.

Por otro lado, en los países de bajos ingresos se ha observado la transición de patologías como opacidades corneales nutricionales y anomalías congénitas a patologías observadas en los países de altos ingresos'.

La prevalencia de enfermedades con etiología genética reportada en el Instituto Mexicano de Oftalmología en el periodo del 1 de enero de 2018 al 31 de octubre de 2019 es del $1.09 \%$, similar a la reportada en otros estudios. Las afecciones más frecuentes son retinosis pigmentaria, glaucoma congénito, distrofia de conos, distrofia corneal, distrofia cono-bastón, distrofia bastón-cono, coloboma de iris y catarata congénita.

El diagnóstico genético basado en métodos de detección de ADN establece la predisposición que tiene un paciente para presentar una patología incluso décadas antes de mostrar manifestaciones clínicas, y además permite detectar la presencia de múltiples alteraciones de forma simultánea ${ }^{4}$, lo que tiene repercusiones positivas en la calidad de vida del paciente y su familia, y se traduce en el estudio de las enfermedades que tienen un origen heredable, como son las distrofias retinianas, el glaucoma congénito, la catarata congénita, el estrabismo hereditario, el daltonismo, las distrofias corneales, la atrofia y las neuropatías ópticas heredadas.

El desarrollo de técnicas de diagnóstico molecular, como la secuenciación de última generación, ha permitido conocer la totalidad del exoma, así como secuencias específicas identificadas como causantes de patología ocular heredada, como degeneraciones retinianas, atrofia óptica y glaucoma ${ }^{5}$. En un estudio publicado por Rim, et al. ${ }^{6}$, la secuenciación de nueva generación se utilizó para el estudio del nistagmo a temprana edad, lo que llevó a la detección de otras alteraciones genéticas.

En 2016 se creó The Genetic Eye Disease Task Force como respuesta a la necesidad que tenía la American Association for Pediatric Ophthalmology and Strabismus (AAPOS). Para iniciar, crearon una encuesta con el fin de evaluar el conocimiento de base de las enfermedades más comunes hereditarias oculares: la evaluación de pacientes, el entendimiento de pruebas genéticas y la habilidad para identificar pacientes con cuadros de origen genético que pudieran ser tratados como parte de enfermedades sistémicas. Se reportaron un total de 264 encuestas respondidas, y el $11.7-34.1 \%$ de los participantes reportaron tener conocimiento y usar en su práctica clínica el diagnóstico genético en sus diferentes modalidades ${ }^{7}$.

En México existen pocos estudios que documenten el conocimiento médico acerca de las técnicas de diagnóstico molecular para la detección de patologías oftalmológicas heredadas. En un estudio realizado por la Escuela de Medicina de la Universidad Panamericana, en el año 2015, se realizó un análisis bioético sobre el 
conocimiento y las actitudes de los oftalmólogos en cuanto al consejo genético para retinoblastoma, el cual reportó que un $73.5 \%$ de ellos se han visto en la necesidad de proporcionar asesoramiento a los pacientes sobre la enfermedad; sin embargo, solo el $26.5 \%$ demostraron tener conocimientos sobre los patrones de herencia del retinoblastoma ${ }^{8}$.

El objetivo de este estudio fue describir los resultados obtenidos en una encuesta realizada a oftalmólogos pertenecientes a la Asociación Mexicana de Oftalmología Pediátrica (AMOP), la Sociedad Latinoamericana de Oftalmología Pediátrica (SOPLA) y la Sociedad Panamericana de Retinopatía del Prematuro (SPROP), sobre el abordaje de patologías de probable origen genético, así como sobre el uso de pruebas diagnósticas, el conocimiento actual y el futuro interés de aprendizaje.

\section{Método}

Se trata de un estudio observacional y descriptivo. El protocolo fue aprobado por el Comité de Investigación del Instituto Mexicano de Oftalmología.

Se aplicó un cuestionario mediante una plataforma electrónica (Google y onlinencuesta), el cual consistió en 24 preguntas abiertas, cerradas y de opción múltiple, y fue compartido con oftalmólogos pertenecientes a la AMOP, la SOPLA y la SPROP por medio de invitaciones electrónicas, en los meses de noviembre y diciembre de 2019. Se especificó en la encuesta que los resultados serían anónimos y solo se utilizarían con fines académicos.

El cuestionario se obtuvo de un estudio realizado por Drack, et al. ${ }^{7}$, con previa autorización, y fue traducido al español, mencionando que el cuestionario original no está validado. Se obtuvieron en total 100 respuestas. Los criterios de selección fueron ser médico oftalmólogo y perteneciente a las sociedades AMOP, SOPLA o SPROP.

Se realizó un análisis estadístico descriptivo de las variables de interés y para cada una se determinaron las prevalencias para la muestra total; los resultados se expresaron como porcentajes.

\section{Resultados}

La encuesta electrónica fue contestada por 100 oftalmólogos pertenecientes a las sociedades antes mencionadas, de los cuales el $79 \%$ se dedican dentro de su práctica clínica a la enseñanza y el $27 \%$ a la investigación. Así mismo, el $56 \%$ ejercen la medicina privada, el $21 \%$ en un hospital oftalmológico y el $35 \%$ en hospitales públicos. De los 100 participantes, el 73\% trabajan tiempo completo y el $27 \%$ medio tiempo.

De los entrevistados se reporta que, en promedio a la semana, la mayoría (70\%) atienden menos de cinco pacientes con patología ocular de probable origen genético, el $15 \%$ de 5 a 10 pacientes, el $4 \%$ más de 11 pacientes y el $11 \%$ refieren no atender este tipo de patologías. Se preguntó si realizaban estudios genéticos a los pacientes en quienes sospechaban un origen genético del padecimiento, como se documenta en la figura 1.

Se interrogó de forma intencionada acerca de las patologías genéticas pediátricas más relevantes en la consulta de un oftalmólogo, de acuerdo con su prevalencia, como son el nistagmo, la catarata juvenil, la catarata congénita y el glaucoma congénito (Tabla 1), siendo la catarata congénita y juvenil la mayormente asociada a una patogénesis genética (95\%).

Del total de los médicos entrevistados, en cuanto a las pruebas usadas para el diagnóstico genético reportaron un grado de conocimientos que varía del $2 \%$ al $27 \%$. El $55 \%$ de los médicos reportaron no estar familiarizados con dichas técnicas, el $18 \%$ no comprenden las pruebas diagnósticas en genética y solo el $1 \%$ las comprenden completamente. Por otro lado, la mayoría reportan tener interés por el aprendizaje y la implementación de dichas pruebas diagnósticas en su práctica clínica diría, como se muestra en la figura 2.

\section{Discusión}

A partir de la información obtenida de los oftalmólogos encuestados, destaca la deficiencia en la práctica clínica en el reconocimiento de patologías de origen genético, así como en su abordaje diagnóstico, pues solo el $27 \%$ de los médicos refieren realizar pruebas diagnósticas, de los cuales el $78 \%$ terminan refiriendo el caso a un especialista en genética.

De acuerdo con el estudio realizado por Drack, et al. ${ }^{7}$, en el que se basó el presente trabajo, el porcentaje más alto en conocimiento basal sobre métodos diagnósticos fue del $34 \%$, en el rubro de análisis de deleciones y duplicaciones, y el más bajo fue en diagnóstico por secuenciación de Sanger, con un $11.7 \%$; el $48 \%$ respondieron no estar familiarizados con dichas técnicas. En constraste, en el presente estudio, el análisis de mutaciones puntuales reportó el nivel de conocimiento más alto $(27 \%)$, y el más bajo lo obtuvo la secuenciación de Sanger (2\%), mientras que el $55 \%$ respondieron no estar familiarizados con ninguna técnica diagnóstica.

Respecto a la asociación con condiciones genéticas, se identificó el nistagmo como de etiología genética en 


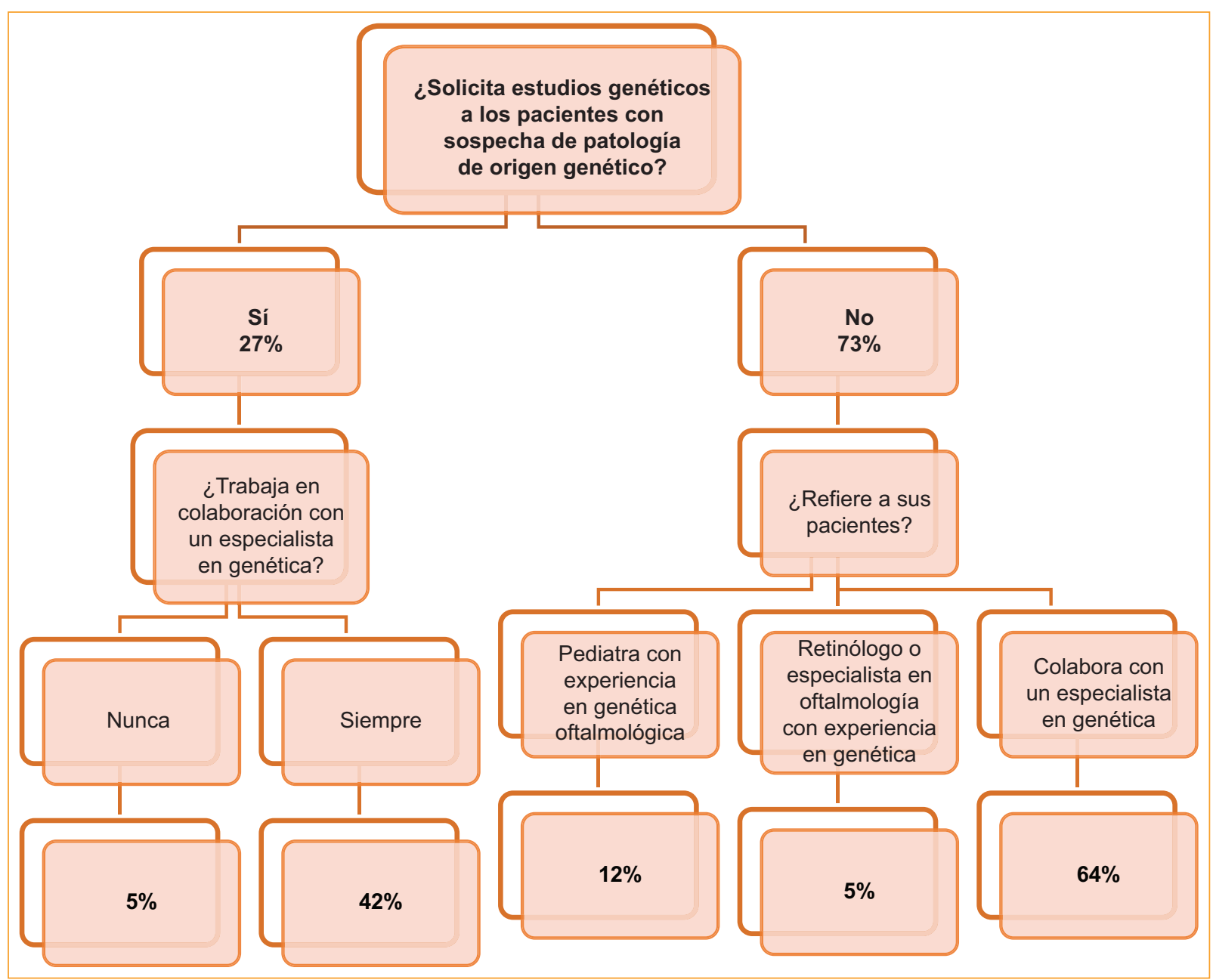

Figura 1. Flujograma del abordaje diagnóstico en pacientes con patologías de probable origen genético en oftalmología pediátrica.

Tabla 1. Principales patologías de probable origen genético en la consulta de oftalmología pediátrica

\begin{tabular}{|l|c|c|c|c|}
\hline & $\begin{array}{c}\text { iEs una patología } \\
\text { de potencial } \\
\text { origen genético? }\end{array}$ & \multicolumn{2}{|c|}{$\begin{array}{c}\text { iRealiza } \\
\text { diagnóstico } \\
\text { genético? }\end{array}$} \\
\cline { 2 - 6 } & Sí & No & Sí & No \\
\hline Nistagmo & $77 \%$ & $23 \%$ & $21 \%$ & $79 \%$ \\
\hline Catarata juvenil/congénita & $95 \%$ & $5 \%$ & $67 \%$ & $33 \%$ \\
\hline Glaucoma congénito & $94 \%$ & $6 \%$ & $35 \%$ & $65 \%$ \\
\hline
\end{tabular}

un $77 \%$, la catarata juvenil/congénita en un $95 \%$ y el glaucoma congénito en un $94 \%$, mientras que en el estudio de Drack, et al. ${ }^{7}$ los médicos identificación en un $75 \%$ el nistagmo, un $95 \%$ la catarata y un $85 \%$ el glaucoma.
Entre los entrevistados, de acuerdo con la práctica clínica, de los que trabajan en un hospital oftalmológico el $61 \%$ respondieron sentirse capacitados para identificar manifestaciones clínicas de condiciones genéticas y hacer referencia oportuna, el $74 \%$ de los que trabajan en un hospital general y el $25 \%$ de los que trabajan en la práctica privada.

El interés de los médicos por aprender sobre genética aplicada en su práctica clínica es alto, con especial interés en la secuenciación completa del exoma (64\%) y en el análisis de mutaciones puntuales en un $61 \%$, mientras que en el estudio de Drack, et al. ${ }^{7}$ el mayor interés en un futuro aprendizaje se reportó para el análisis de mutaciones puntuales (73.5\%) y la secuenciación de nueva generación (72.7\%).

Entre las sugerencias de los oftalmólogos encuestados, se propone profundizar en conocimientos 


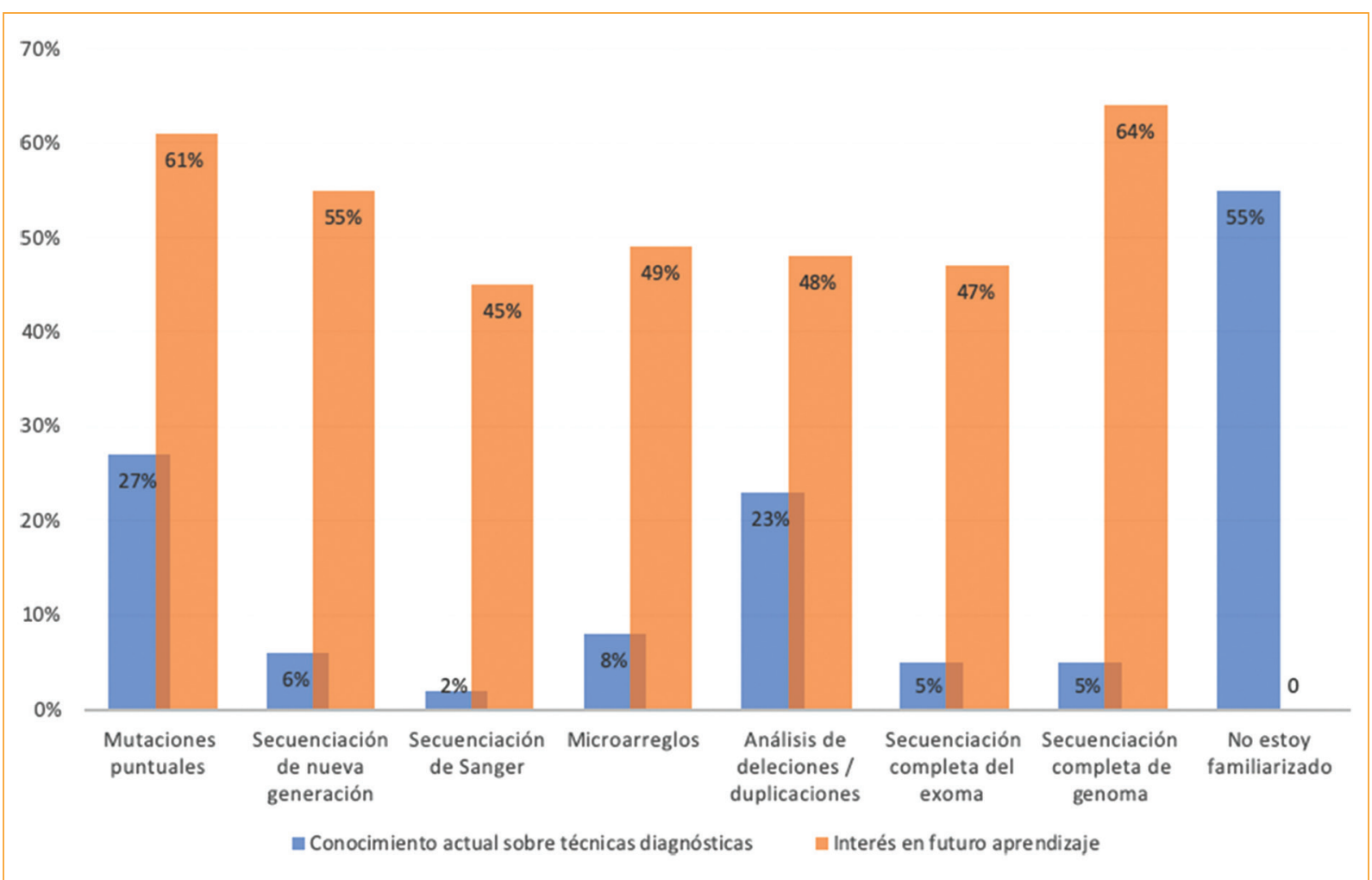

Figura 2. Conocimiento actual y futuro interés de aprendizaje sobre técnicas de diagnóstico genético.

genéticos mediante la creación de cursos en línea sobre genética aplicada, congresos enfocados en genética y la colaboración con genetistas para otorgar cursos.

Una de las limitaciones del estudio es que el cuestionario no está validado; sin embargo, refleja la importancia de prestar atención al abordaje genético del paciente oftalmológico.

La medición del conocimiento médico sobre patologías de origen genético permite identificar las áreas de oportunidad dentro de la oftalmología, así como promover el aprendizaje en genética de patologías oculares, tanto del diagnóstico como del tratamiento y el pronóstico, y poder otorgar asesoramiento como parte de un manejo multidisciplinario.

El impacto que tiene el diagnóstico genético en la vida del paciente puede significar su reincorporación a la sociedad y prevenir complicaciones al actuar de forma precoz. Se busca evitar la recurrencia en la familia, así como asesorar sobre la aplicación de pruebas de diagnóstico molecular para los miembros que podrían estar potencialmente afectados. Se refleja además la importancia de la realización de algoritmos diagnósticos aplicables a población mexicana, para de este modo brindar tratamiento específico y personalizado basado en las alteraciones genéticas más frecuentes.

\section{Conclusiones}

Concluimos que, en la práctica clínica de los oftalmólogos pediátricos encuestados, existe una deficiencia en el reconocimiento de patologías de origen genético, así como en su abordaje diagnóstico. Sin embargo, la mayoría de los oftalmólogos muestran gran interés por aprender sobre genética aplicada en su práctica diaria, en especial la secuenciación completa del exoma y el análisis de mutaciones puntuales.

\section{Conflicto de intereses}

Se declara que no existe ningún conflicto de intereses por parte de los autores ni del Instituto Mexicano de Oftalmología, por lo que los resultados no se vieron influenciados de forma alguna. 


\section{Responsabilidades éticas}

Protección de personas y animales. Los autores declaran que para esta investigación no se han realizado experimentos en seres humanos ni en animales.

Confidencialidad de los datos. Los autores declaran que han seguido los protocolos de su centro de trabajo sobre la publicación de datos de pacientes.

Derecho a la privacidad y consentimiento informado. En el presente trabajo no se usaron datos de pacientes, solo una encuesta por medio electrónico, en la que se especificó que la información compartida sería únicamente para fines de investigación. No se incluyeron datos personales para mantener el anonimato de los médicos participantes.

\section{Bibliografía}

1. Solebo A, Teoh L, Rahi J. Epidemiology of blindness in children. Arch Dis Child. 2017;102:853-7.

2. Bunce $C$, Wormald R. Leading causes of certification for blindness and partial sight in England and Wales. BMC Public Health. 2006;6:58.

3. Cleveland Clinic. Inherited eye disease. (Consultado el 31 de diciembre de 2019.) Disponible en: https://my.clevelandclinic.org/health/diseases/17130-inherited-eye-disease.

4. Stone E, Aldave A, Drack AV, MacCumber M, Sheffield V, Traboulsi E, et al. Recommendations for genetic testing of inherited eye diseases. Ophthalmology. 2012;119:2408-10.

5. Consugar M, Navarro-Gómez D, Place E, Bujakowska K, Sousa M, Fonseca-Kelly Z. Panel-based genetic diagnostic testing for inherited eye diseases is highly accurate and reproducible and more sensitive for variant detection, than exome sequencing. Genet Med. 2014;17:253-5.

6. Rim JH, Lee ST, Gee HY, Lee BJ, Choi JR, Park HW, et al. Accuracy of next-generation sequencing for molecular diagnosis in patients with infantile nystagmus syndrome. JAMA Ophthalmol. 2017;135:1376-85.

7. Drack A, Miraldi V, Alcorn D. Survey of practice patterns for the management of ophthalmic genetic disorders among AAPOS members: report by the AAPOS Genetic Eye Disease Task Force. J AAPOS. 2019;23:223-6.

8. Morelos $\mathrm{P}$, Casas $\mathrm{M}$, Juárez J, Villa A. Conocimiento y actitudes de oftalmólogos mexicanos acerca del consejo genético para retinoblastoma: análisis bioético. Rev Mex Oftalmol. 2016;90:276-83. 